\title{
Behavioural and Electrophysiological Correlates of Aided Performance in Individuals with Late Onset Auditory Neuropathy Spectrum Disorder: A Review
}

\author{
Jijo Pottakkal Mathai \\ JSS Institute of Speech and Hearing, Kelageri, Dharwad, India
}

Received February 17, 2018

Revised April 28, 2018

Accepted July 1, 2018

Address for correspondence Jijo Pottakkal Mathai, $\mathrm{PhD}$

JSS Institute of Speech and Hearing, Near Tapovana,

Sri Shivarathreeshwara Nagar,

Kelageri, Dharwad 580007, India

Tel +919620467904

E-mail jijoaudio@gmail.com
Hearing aids are one of the rehabilitative options for individuals with late onset auditory neuropathy spectrum disorder (ANSD). There is a growing body of evidence that supports the effectiveness of hearing aids among these individuals. The objective of the study was to review the behavioural and electrophysiological factors that determine the effectiveness of amplification in individuals with late onset ANSD. An intensive search was carried out using the PubMed, SpringerLink and Google Scholar databases using the following keywords: auditory neuropathy, late onset ANSD, hearing aids and amplification. There were both retrospective and experimental research studies that showed aided improvement in individuals with late onset ANSD. The aided improvement in these studies has been attributed to preserved neural synchrony. Hence, it was concluded that, hearing aids are effective in improving speech perception in a sub-group of individuals with late onset ANSD. Measures that evaluate neural synchrony are promising tools to identify the sub-group of such individuals. Speech identification scores at higher sensation levels and cortical potentials are the two clinical measures that indicate aided performance in individuals with late onset ANSD.

J Audiol Otol 2018;22(4):171-177

KEY WORDS: Auditory neuropathy spectrum disorder · Hearing aids . Speech identification score $\cdot$ Cortical potential.

\section{Introduction}

Auditory neuropathy spectrum disorder (ANSD) is a form of sensori-neural hearing loss (SNHL) that leads to impaired neural synchrony but preserved outer hair cell functioning. The age of onset of ANSD has been reported predominantly in two distinct age groups: infancy and adolescence or early adulthood [1-6]. Individuals with ANSD exhibit diverse site of lesion and perceptual deficits that often challenge their rehabilitation.

Hearing aids are one of the rehabilitative options, often reported to be effective in the paediatric-group of ANSD [7-9]. However, usefulness of hearing aids is often limited in those having late onset ANSD [6,10-17]. A few among such studies

This is an Open Access article distributed under the terms of the Creative Commons Attribution Non-Commercial License (https://creativecommons.org/licenses/by-nc/4.0/) which permits unrestricted non-commercial use, distribution, and reproduction in any medium, provided the original work is properly cited. that reported insufficient aided improvement in late onset ANSD were narrative reports or clinical experiences, without giving any measurable information on aided performance [1012]. Further, a few studies were carried out on clients having rapidly advancing, late onset ANSD hence, their results were inconclusive [13,14,17].

There is a growing body of evidence that supports the effectiveness of amplification in those with late onset ANSD. In a retrospective study, Shetty, et al. [18] compared aided improvement in speech perception in 83 individuals with SNHL and 82 individuals with late onset ANSD. The findings revealed that, individuals with late onset ANSD had aided improvement up to $20 \%$. However, individuals with SNHL showed aided improvement that ranged from $22 \%$ to $88 \%$. Unlike SNHL, the aided improvement in individuals with late onset ANSD was unrelated to the degree of hearing loss. The study concluded that, the use hearing aids in individuals with late onset ANSD was less effective compared to SNHL. 
Lima, et al. [19] monitored speech recognition threshold (SRT) of a client with late onset ANSD in quiet and noisy environments. The client had moderate hearing loss in the right ear and normal threshold in low frequencies with a slight high frequency sloping in the left ear. Hearing aid was fitted in the right ear. It was reported that sentence recognition threshold in both quiet and noisy environments improved within the first 3 months of hearing aid use. Moreover, administration of Hearing Handicap Inventory before hearing aid adaptation showed 40 points reduction in hearing handicap. Similarly, Dell'Aringa, et al. [20] reported aided performance in a 32 years old man having Guillain-Barré syndrome. The client had bilateral severe SNHL and other audiological manifestations associated with ANSD. The use of hearing aid in this individual led to improved aided threshold and perception of polysyllabic words. Speech perception score improved from $12 \%$ in the unaided condition to $44 \%$ in the aided condition, even though with lip reading. Although these single case studies supported the effectiveness of hearing aids, the reason for the aided improvement was not stated.

Recently, there were a few investigations that used behavioural or electrophysiological measures to identify the subgroups of individuals who may benefit from hearing aids. Till date there were no systematic reviews on aided performance in individuals with late onset ANSD. Therefore, this review of literature is an attempt to provide an overview of such studies that supported the effectiveness of amplification in individuals with late onset ANSD. The review also compares the findings of recent studies on aided performance among individuals with late onset ANSD and highlights the factors that lead to aided improvement. It will be useful for practicing audiologist in identifying the sub-group of individuals with late onset ANSD who may benefit from hearing aids; and prescribing the appropriate hearing aids that fit their condition.

\section{Methods}

A systematic and orderly review was carried out to accumulate information on the aided performance in individuals with late onset ANSD. An intensive search was carried out in the following database: PubMed, SpringerLink and Google Scholar using the following keywords: auditory neuropathy, late onset ANSD, hearing aids and amplification. The scientific literatures published between 1998 to 2017 were considered for the review. Original articles, reviews and case reports on individuals with late onset ANSD were included in the study. There were more than 600 research publications related to auditory neuropathy available in the database. Hence, below mentioned criteria were used to select those articles that were relevant to the review.

\section{Selection criteria}

The studies that investigated the aided performance in individuals with late onset ANSD were included in the systematic review. Thus, investigations in paediatric ANSD, though they described aided performance, were excluded from the study. The aided performance in these studies was evaluated using behavioural tests such as speech identification scores (SIS), temporal processing abilities. Further, studies that used late latency response (LLR) as an electrophysiological measure to evaluate aided performance were also included. Articles that did not meet the inclusion criteria were excluded.

\section{Data collection}

Till date there were only 10 studies that investigated the role of behavioural or electrophysiological findings on aided performance. Among the 10 studies, 2 of them were retrospective studies and the remaining 8 were experimental research. Table 1 and 2 depict the summary of recent studies conducted on behavioural and electrophysiological correlates of aided improvement in late onset ANSD.

\section{Results}

\section{Behavioural test findings and aided performance}

In a retrospective study, Mathai and Yathiraj [21] investigated the relation between audiological findings and aided improvement in 64 clients with late onset ANSD. The results showed improvement in speech perception in 39 out of the 104 ears while using hearing aids. However, the mean aided improvement was only $19.1 \%$ with a large SD of $14 \%$ showing the minimal and diverse aided performance. There was a strong positive correlation between aided improvement and SIS measured at $40 \mathrm{~dB}$ SL (ref to SRT). However, there was no relation between aided improvement and unaided SIS or pure-tone average (PTA). It was reported that relatively better SIS in a few individuals could be due to preserved neural synchrony that made up for distortion in spectral cues that occurred at higher presentation level (40 dB SL). In contrast, individuals who had poor SIS at higher level (40 dB SL ref to SRT) might have had poor neural synchrony causing poor speech perception at higher level of presentation. Hence, the execution of performance-intensity function was recommended for individuals with ANSD in order to identify the subgroup that might show aided improvement.

Similarly, Narne, et al. [22] in a retrospective study, investigated gender difference in audiological findings and aided performance in 255 individuals with ANSD. Majority of the 


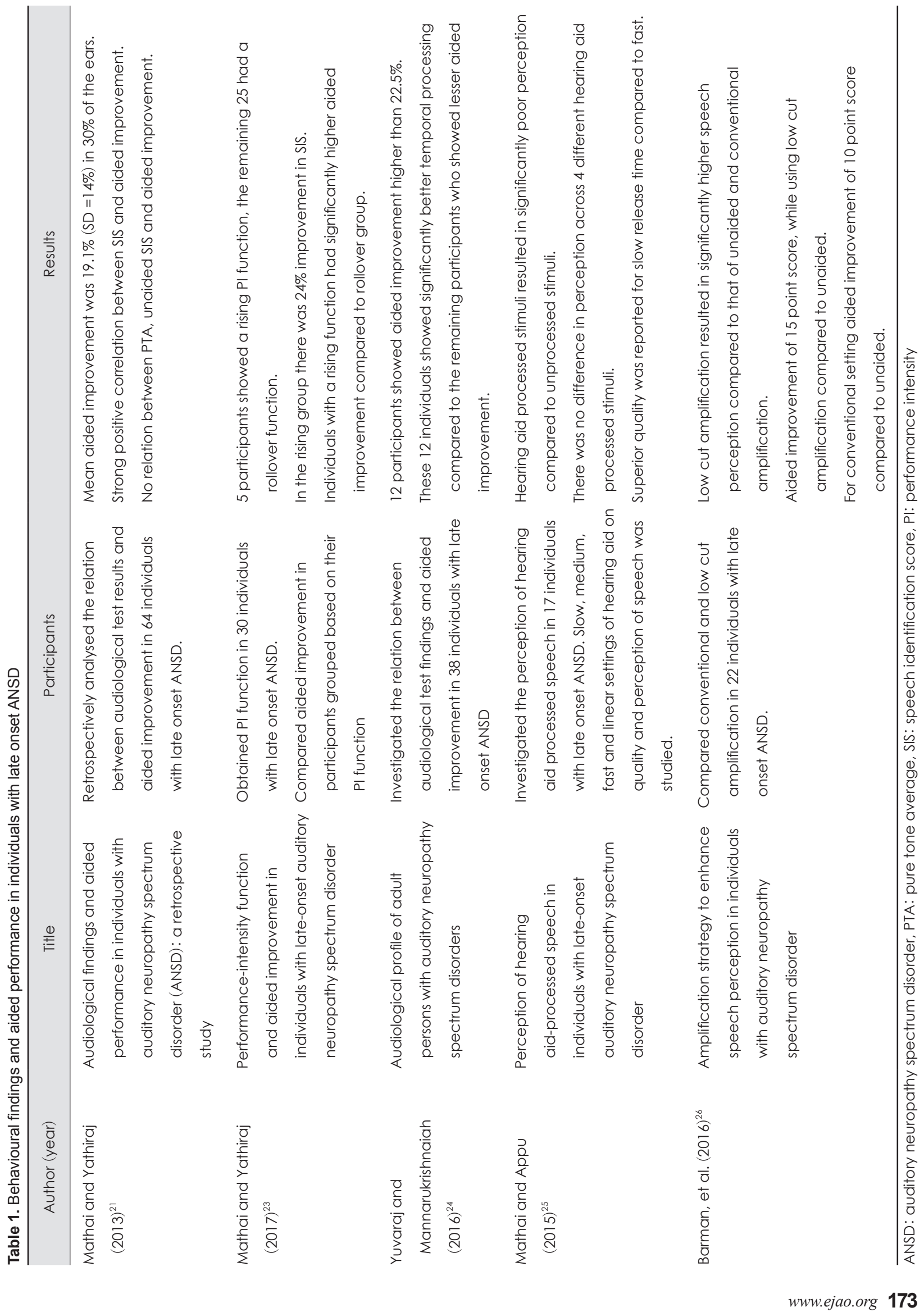


subjects developed the condition after 10 years; indicating late onset ANSD. The study observed gender difference in degree of hearing loss, audiometric configuration, speech perception and hearing aid benefit. It was reported that all the participants who benefitted from hearing aids had SIS higher than $50 \%$.

Mathai and Yathiraj [23] also investigated the effect of intensity on speech perception and aided performance in 30 individuals with late onset ANSD. Performance intensity (PI) function was obtained using phonemically balanced words presented at different sensation levels showed two distinct patterns. There were $25(83.3 \%)$ participants who had a rollover function where the SIS reduced with increasing presentation levels. However, remaining 5 (16.7\%) participants had a rising function where speech identification improved with presentation levels. Accordingly, aided scores in the rising group were significantly higher than the unaided scores; whereas in the rollover group there was no such difference between unaided and aided scores found. Authors attributed that, individuals who showed a rising function might have preserved neural synchrony that helped them to perceive speech at higher presentation levels. In contrast, poor neural synchrony in the rollover group lead to diminished speech perception at higher levels. It was concluded that obtaining a complete PI function can be an indicator of aided performance in individuals with late onset ANSD. However, usefulness of hearing aids in daily listening needs is questionable as the aided performance was not studied in noisy situations. Moreover, mean improvement in the aided condition was only $24 \%$ in the rising group.

Yuvaraj and Mannarukrishnaiah [24] investigated aided improvement in speech identification in 38 individuals with late onset ANSD. Further, they estimated the relation between audiological findings (PTA, SIS, and temporal processing) and aided benefit. The clients showed an aided improvement in speech identification ranging from $5 \%$ to $50 \%$ over the unaided. Correlation analysis showed significant correlation between modulation detection threshold and aided benefit. Authors concluded that those having better temporal processing can overcome the temporal distortion resulting from hearing aids. Thus, temporal processing measured using modulation detection could be an indicator of aided performance in individuals with late onset ANSD.

Mathai and Appu [25] studied perception of hearing aid processed speech in 17 individuals with late onset ANSD. Phonemically balanced words were processed using a hearing aid with three different compression time settings (slow, fast, and medium) and a linear processing strategy. Their results revealed no significant difference in speech perception across the four processing strategies. This was attributed to identical temporal envelope of the stimuli in all the four hearing aid settings. However, superior quality was noted when the stimuli were processed using a slow release time compared to fast. It was concluded that though speech perception scores did not vary across settings, hearing aids with fast release time should not be recommended for individuals with ANSD as there was poor quality rating. However, authors reported that results may be interpreted with caution as they utilised hearing aid processed stimuli rather than individually programmed hearing aids.

Barman, et al. [26] investigated the effect of conventional standard NAL-NL1 prescriptive approach and low-cut amplification strategy on aided speech identification in 22 participants with late onset ANSD. They hypothesized that, in case of conventional hearing aid amplification, low frequencies are amplified more due to reverse sloping or peaked audiogram configurations seen in most of the clients. Thus, the conventional method of providing amplification could unnecessarily amplify low frequencies that may not be used by individuals with ANSD. They observed that this low frequency amplification may affect speech perception because of upward spread of masking. It was found that low cut amplification resulted in significantly higher speech perception compared to unaided and standard amplification. This was attributed to reduced low frequency content in the low cut amplification strategy that eliminated upward spread of masking.

Similarly, Prabhu and Barman [27] compared aided benefit in individuals with late onset ANSD using receiver in the canal (RIC) and behind the ear (BTE) hearing aids. Aided benefit was compared between these two hearing aids using conventional and low cut amplification. There were 20 participants having bilateral mild to moderate degree of hearing loss involved in the study. They found that aided benefit using low cut amplification was significantly higher than conventional amplification for both RIC and BTE hearing aids. Further, the aided benefit was significantly higher for RIC hearing aids compared to that of BTE. Higher speech perception in ANSD using RIC hearing aids over BTE has been attributed to better sound quality and naturalness of speech using the former type of hearing aids.

Prabhu and Barman [28] compared aided benefit using conventional, low-cut modified amplification and channelfree hearing aids in 25 individuals with late onset ANSD. All the participants had acquired ANSD and bilateral moderate to severe degree of hearing loss. Based on SIS participants were further divided into good performers (above 50\% score) and poor performers (below 50\% score). They found that good performers showed significantly higher aided benefit compared to conventional and low-cut modified amplifica- 


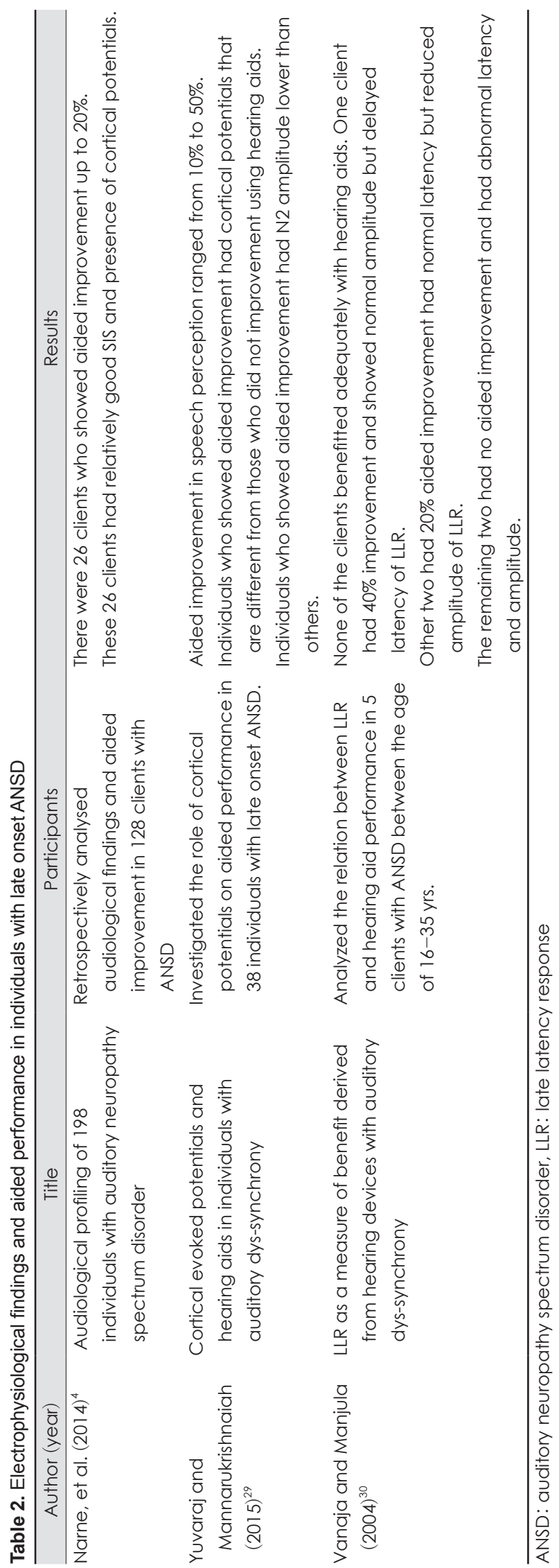

tion. Among poor performers though there was no difference between channel-free and low-cut modified amplification, aided benefit in these two conditions were significantly better than conventional amplification. Aided benefit in channel free hearing aids is ascribed to lack of multiple channels in them that in turn could have lead to lesser distortion. They concluded that channel-free hearing aids and low-cut modified amplification can be used as an efficient technique to improve aided speech perception in individuals with late onset ANSD.

It is clear from the above studies that individuals with late onset ANSD showed wide range of aided improvement. This is evidenced by a large standard deviation in the aided improvement. It is clear from the above studies that behavioural measures such as SIS and modulation detection thresholds were good predictors of aided improvement. However, degree of hearing loss and unaided SIS were not predictors of aided improvement.

\section{Electrophysiological findings and aided performance}

In a retrospective study, Narne, et al. [4] described audiological findings and hearing aid benefit in 198 individuals with ANSD. It was noticed that $82 \%$ of the clients had late onset ANSD. Among the 128 clients who had undergone hearing aid trial, 39 were recommended to use hearing aids. It was noted that 26 out of the 39 clients had $20 \%$ improvement in speech identification in the aided condition over the unaided. In the remaining 13 clients hearing aid was helpful only for auditory awareness. Cortical auditory evoked potentials were present in those 26 clients who demonstrated some functional benefits using hearing aids. The presence of cortical potentials in these individuals was considered to suggest preserved neural synchrony in them that was substantiated by good SIS. Consequently, it was reasoned that aided speech perception improvement in individuals with ANSD depends on neural synchrony.

Yuvaraj and Mannarukrishnaiah [29] investigated the role of cortical potentials on aided performance in 38 individuals with late onset ANSD. Participants with ANSD were grouped into 4 subgroups based on their aided performance. Group 1 had significantly higher aided benefit compared to the other three groups. Results revealed that P1 latency and N2 amplitude of group 1 were significantly different from other groups. Authors reported that significantly lowest N2 amplitude in group 1 reflect greater inhibitory ability to suppress the unwanted noise. This might have helped these individuals to overcome the disadvantages of hearing aid processing. They also observed an average aided improvement of $14 \%$ in their participants.

Vanaja and Manjula [30] investigated the relation between 
LLR and aided performance in 5 clients with late onset ANSD. Each individual was tested using an analogue hearing aid having gain matched using the NAL-R prescriptive formula, fitted monaurally. Only one participant showed aided benefit up to $40 \%$ whereas two clients had only a minimal benefit (20\%) and the remaining two clients had no improvement. It was observed that the client with typical LLR amplitude had maximum aided benefit, whereas those who had lower amplitude had lesser advantage from hearing aids. The two clients who did not benefit from hearing aids had no replicable LLR. The authors concluded that in participants with anomalous auditory brainstem response (ABR) and typical LLR, the functional deficiency at the level of the brainstem is remunerated by the cortical mechanisms. In contrast, speech understanding would be more affected in those with abnormal ABR and abnormal LLR, because of deficits in the cortical as well as brainstem functioning. Similarly, Narne, et al. [22] reported aided benefits in individuals with late onset ANSD in whom LLR was present.

\section{Conclusion}

It can be seen from the above studies that a couple of individuals with late onset ANSD showed improvement in speech perception using hearing aids. However, the extent of aided improvement in majority of the clients is limited. Investigations by Mathai and Yathiraj [23] as well as Yuvaraj and Mannarukrishnaiah [24] showed aided improvement above 20\%, whereas other studies have shown lesser aided improvement. Relatively higher aided improvement in a few participants was attributed to preserved neural synchrony. A rising performance intensity for phonetically balanced (PIPB) function or preserved temporal processing abilities were indications of preserved neural synchrony. Hence, behavioural measures such as PIPB function or measures of temporal resolution can be utilised to predict aided improvement in individuals with late onset ANSD. Those individuals having poor neural synchrony indicated by flat or rollover PIPB function or poor temporal resolution may not show an aided improvement comparable to those individuals having preserved neural synchrony.

Among children, presence of cortical potentials has been found to be an indication of aided improvement [9]. Similarly, in individuals with late onset ANSD, there was relation between presence of cortical potentials and aided improvement. Cortical potentials are reported to be an electrophysiological measure of neural synchrony. Hence, it can be concluded that presence of cortical potentials or relatively better speech identification scores at higher sensation levels are the two tests for neural synchrony that can be used to predict the aided improvement.

All the above studies that investigated aided performance in individuals with late onset ANSD were conducted quietlaboratory set-up. None of them have reported information on hearing aid usage in day to day communication. As most of the listening environments are noisy and individuals with ANSD exhibit poor speech perception in noise, there is a need to investigate usefulness of amplification in real life situations.

In conclusion, there is a sub-group of individuals with late onset ANSD who showed improvement in speech perception while using hearing aids. SIS at higher sensation levels or cortical potentials are the two promising tools that measures neural synchrony in these individuals and may predict their aided performance. Usefulness of hearing aids in everyday communication is still questioned as the extent of aided improvement is limited in majority of the clients and aided speech perception in noise is not well studied.

\section{Acknowledgments}

Author would like to thank the All India Institute of Speech and Hearing, Mysore, where the study was carried out. Sincere thanks to Mr. Stephen Nanbur, Department of Nursing Sciences, Faculty of Medical Sciences, University of Jos, Nigeria for his comments to improve the language.

\section{Conflicts of interest}

The author has no financial conflicts of interest.

\section{REFERENCES}

1) Berlin CI, Hood LJ, Morlet T, Wilensky D, Li L, Mattingly KR, et al. Multi-site diagnosis and management of 260 patients with auditory neuropathy/dys-synchrony (auditory neuropathy spectrum disorder). Int J Audiol 2010;49:30-43.

2) Mathai JP, Yathiraj A. Audiological characteristics and duration of the disorder in individuals with auditory neuropathy spectrum disorder (ANSD)- a retrospective study. J Indian Speech Language Hearing Assoc 2012;26:17-26.

3) Kumar UA, Jayaram MM. Prevalence and audiological characteristics in individuals with auditory neuropathy/auditory dys-synchrony. Int J Audiol 2006;45:360-6.

4) Narne VK, Prabhu P, Chandan HS, Deepthi M. Audiological profiling of 198 individuals with auditory neuropathy spectrum disorder. Hearing Balance Commun 2014;12:112-20.

5) Sininger Y, Starr A. Auditory neuropathy: a new perspective on hearing disorders. 1st ed. San Diego: Singular Thomson Learning;2001.

6) Starr A, Picton TW, Sininger Y, Hood LJ, Berlin CI. Auditory neuropathy. Brain 1996;119(Pt 3):741-53.

7) Rance G, Barker EJ, Sarant JZ, Ching TY. Receptive language and speech production in children with auditory neuropathy/dyssynchrony type hearing loss. Ear Hear 2007;28:694-702.

8) Rance G, Beer DE, Cone-Wesson B, Shepherd RK, Dowell RC, King AM, et al. Clinical findings for a group of infants and young children with auditory neuropathy. Ear Hear 1999;20:238-52.

9) Rance G, Cone-Wesson B, Wunderlich J, Dowell R. Speech perception and cortical event related potentials in children with auditory neuropathy. Ear Hear 2002;23:239-53.

10) Berlin CI, Hood LJ, Cecola RP, Jackson DF, Szabo P. Does type I af- 
ferent neuron dysfunction reveal itself through lack of efferent suppression? Hear Res 1993;65:40-50.

11) Berlin CI. Auditory neuropathy: using OAEs and ABRs from screening to management. Semin Hear 1999;20:307-14.

12) Berlin CI, Li L, Hood LJ, Morlet T, Rose K, Brashears S. Auditory neuropathy/dys-synchrony: after the diagnosis, then what? Semin Hear 2002;23:209-14.

13) Miyamoto RT, Kirk KI, Renshaw J, Hussain D. Cochlear implantation in auditory neuropathy. Laryngoscope 1999;109(2 Pt 1):181-5.

14) Shallop JK, Peterson A, Facer GW, Fabry LB, Driscoll CL. Cochlear implants in five cases of auditory neuropathy: postoperative findings and progress. Laryngoscope 2001;111(4 Pt 1):555-62.

15) Sininger YS, Hood LJ, Starr A, Berlin CI, Picton TW. Hearing loss due to auditory neuropathy. Audiol Today 1995;7:10-3.

16) Dorman MF, Studdert-Kennedy M, Raphael LJ. Stop-consonant recognition: release bursts and formant transitions as functionally equivalent, context-dependent cues. Percep Psychophys 1977;22:109-22.

17) Widen JE, Ferraro JA, Trouba SE. Progressive neural hearing impairment: case report. J Am Acad Audiol 1995;6:217-24.

18) Shetty HN, Mathai JP, Uppunda AK. Disability certificate for individuals with hearing impairment-time to rethink. Indian J Otol 2017; 23:7-12.

19) Lima AP, Mantello EB, Anastasio AR. Monitoring the hearing handicap and the recognition threshold of sentences of a patient with unilateral auditory neuropathy spectrum disorder with use of a hearing aid. Int Arch Otorhinolaryngol 2016;20:185-8

20) Dell'Aringa AHB, Esteves MCBN, Dell'Aringa AR, Arruda GV. Hearing aid fitting results in a case of a patient with auditory neuropathy. Int Arch Otorhinolaryngol 2009;13:107-10.

21) Mathai JP, Yathiraj A. Audiological findings and aided performance in auditory neuropathy spectrum disorder: a retrospective study. J Hear Sci 2013;3:18-26.
22) Narne VK, Prabhu P, Chandan HS, Deepthi M. Gender differences in audiological findings and hearing aid benefit in 255 individuals with auditory neuropathy spectrum disorder: a retrospective study. J Am Acad Audiol 2016;27:839-45.

23) Mathai JP, Yathiraj A. Performance-intensity function and aided improvement in individuals with late-onset auditory neuropathy spectrum disorder. Ear Hear 2017;38:e109-17.

24) Yuvaraj P, Mannarukrishnaiah J. Audiological profile of adult persons with auditory neuropathy spectrum disorders. J Audiol Otol 2016;20: 158-67.

25) Mathai JP, Appu S. Perception of hearing aid-processed speech in individuals with late-onset auditory neuropathy spectrum disorder. J Am Acad Audiol 2015;26:815-23.

26) Barman A, Sinha SK, Prabhu P. Amplification strategy to enhance speech perception in individuals with auditory neuropathy spectrum disorder. Hear Balance Commun 2016;14:25-35.

27) Prabhu P, Barman A. Effectiveness of low cut modified amplification using receiver in the canal hearing aid in individuals with auditory neuropathy spectrum disorder. Int Arch Otorhinolaryngol 2017;21: 243-9.

28) Prabhu P, Barman A. Effectiveness of low-cut modified amplification strategy and channel-free hearing aid in individuals with auditory neuropathy spectrum disorder. Int J Audiol 2017;56:759-66.

29) Yuvaraj P, Mannarukrishnaiah J. Cortical evoked potentials and hearing aids in individuals with auditory dys-synchrony. J Int Adv Otol 2015;11:236-42.

30) Vanaja CS, Manjula P. LLR as a measure of benefit derived from hearing devices in clients with auditory dyssynchrony. In: Shivashankar N, Shashikala HR (editors). Auditory Neuropathy: Compilation of Seminar papers. Bangalore: National Institute of Mental Health and Neuro Sciences;2004. p.136-46. 